\title{
Doubly diffracted ray from a hard quarterplane
}

\section{Albertsen, Niels Christian}

Published in:

IEEE Transactions on Antennas and Propagation

Link to article, DOI:

$10.1109 / 8.855495$

Publication date:

2000

Document Version

Publisher's PDF, also known as Version of record

Link back to DTU Orbit

Citation (APA):

Albertsen, N. C. (2000). Doubly diffracted ray from a hard quarterplane. IEEE Transactions on Antennas and Propagation, 48(5), 764-767. https://doi.org/10.1109/8.855495

\section{General rights}

Copyright and moral rights for the publications made accessible in the public portal are retained by the authors and/or other copyright owners and it is a condition of accessing publications that users recognise and abide by the legal requirements associated with these rights.

- Users may download and print one copy of any publication from the public portal for the purpose of private study or research.

- You may not further distribute the material or use it for any profit-making activity or commercial gain

- You may freely distribute the URL identifying the publication in the public portal

If you believe that this document breaches copyright please contact us providing details, and we will remove access to the work immediately and investigate your claim 


\title{
Doubly Diffracted Ray from a Hard Quarterplane
}

\author{
N. Chr. Albertsen
}

\begin{abstract}
The scattering of the electromagnetic field from a half wave dipole source around a quarterplane can be calculated from the solutions to two scalar problems, one with a soft quarterplane and one with a hard quarterplane. In both cases, a doubly diffracted ray may exist, but only in the case of the hard quarterplane does this present a problem. The paper develops the necessary transition functions for the diffraction coefficients from the exact wave solution.
\end{abstract}

Index Terms-Electromagnetic scattering, geometrical theory of diffraction, scattering near a vertex.

\section{INTRODUCTION}

$\mathbf{T}$ HE electromagnetic field scattered by a quarterplane was derived in [1] for a halfwave dipole source. Here it was shown that the $\theta$ and $\phi$ components of the scattered field could be found on the far-field sphere through quadrature formulas involving the scattered field from two properly chosen scalar problems, where the perfectly conducting quarterplane is replaced by a soft or hard quarterplane, respectively. The theory is valid for any position of the source, but for the numerical example it was necessary to choose a position that excluded the existence of doubly diffracted rays, since they would cause the vertex diffraction coefficient for the hard quarterplane to have a singularity. In the case of the soft quarterplane, the doubly diffracted rays would not cause a problem since they would contribute only through slope diffracted components, which are not included in the present analysis. The present paper considers the calculation of the scattered field from a hard quarterplane in the case where a doubly diffracted ray exists (see Fig. 1). Here the field from $P$ scattered by the quarterplane consists of a reflected ray, two (singly) edge diffracted rays, a vertex diffracted ray, and one doubly diffracted edge ray from $P$ via $d_{1}$ and $d_{2}$ to the far field. When the distance between the two diffraction points reaches zero, the amplitude of this ray becomes singular, as does that of the vertex diffracted from $O$. This situation will occur when either the $x$ or $y$ coordinate of $P$ or both are positive and the far-field observation point lies on the Keller cone for the doubly diffracted ray when $d_{1}$ and $d_{2}$ coalesce at $O$. A transition function correcting this problem was first considered in [2] and [3], where it is was derived from an approximate quadrature form, based on an assumption of mutual interaction between the two edges and applicable to any angular sector. In the particular case of a hard quarterplane, the exact quadrature form for the scattered field is known, however, and this will be used to derive a transition function for the $90^{\circ}$ angular sector in the following.

Manuscript received October 2, 1998; revised December 16, 1999. The author is with the Department of Mathematical Modeling, Technical University of Denmark, Lyngby DK-2800, Denmark (email: nca@imm.dtu.dk).

Publisher Item Identifier S 0018-926X(00)04378-7.

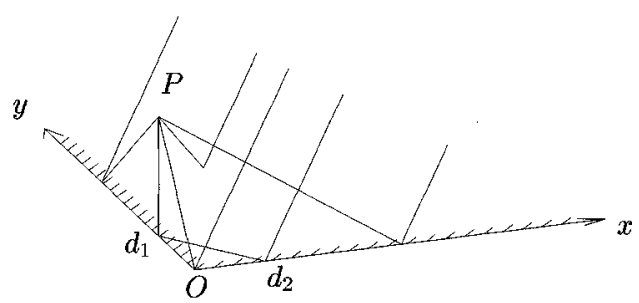

Fig. 1. Quarterplane geometry.

\section{FIELD EXPRESSION}

Since the problem satisfies the requirements of reciprocity, the scattered near field due to an incident plane wave will be considered. Following the procedure of Radlow [4], the scattered field at $P(x, y, z)$ from a hard quarterplane, lying in the first quadrant of the $x-y$ plane and illuminated by a plane wave, incident from the direction $(\theta, \phi)=\left(\theta_{0}, \phi_{0}\right)$ with unit amplitude, time dependence $e^{-i \omega t}$ and wavenumber $k$ can be expressed as

$$
\psi(x, y, z)=\int_{-\infty}^{\infty} d \mu \int_{-\infty}^{\infty} F(\mu, \lambda) e^{i g(\mu, \lambda)} d \lambda
$$

where

$$
\begin{gathered}
F(\mu, \lambda)=-\frac{k \cos \theta_{0} M^{++}\left(\mu, k_{2}\right) M^{++}\left(k_{1}, \lambda\right)}{(2 \pi)^{2} M^{++}\left(-k_{1},-k_{2}\right) M^{++}(\mu, \lambda)} \\
\times \frac{\left[\left(\mu-k_{1}\right)\left(\lambda-k_{2}\right)\right]^{-1}}{\sqrt{k_{t 1}+\lambda} \sqrt{k_{t 2}+\mu}} \\
g(\mu, \lambda)=\gamma z-\mu x-\lambda y \\
\gamma=\sqrt{k^{2}-\mu^{2}-\lambda^{2}} \\
k_{1}=k \sin \theta_{0} \cos \phi_{0}, \quad k_{2}=k \sin \theta_{0} \sin \phi_{0} \\
k_{t 1}=\sqrt{k^{2}-k_{1}^{2}}, \quad k_{t 2}=\sqrt{k^{2}-k_{2}^{2}} .
\end{gathered}
$$

The function $M^{++}(\mu, \lambda)$ is obtained by two Wiener-Hopf factorizations of $\gamma$. It can be expressed by standard special functions, and is defined in [1, Appendix]. The singularities of the integrand in (1) are illustrated in Fig. 2. The pole lines and branch lines are due to the singularities at $\mu=k_{1}, \lambda=k_{2}, \mu=-k_{t 2}$ and $\lambda=-k_{t 1}$. The branch points along the quarter circle in the 
3rd quadrant is due to the presence of $M^{++}(\mu, \lambda)$ in the denominator of (2).

The integral (1) can be evaluated asymptotically as follows (cf. [5]): Consider $\mu$ as a parameter and perform a stationary phase expansion with respect to $\lambda$, then perform a stationary phase expansion with respect to $\mu$ along the dashed curve in Fig. 2 passing through $(\mu, \lambda)=( \pm k, 0)$. Alternatively, the evaluations can be done in the opposite order with $\lambda$ following the dashed curve passing through $(\mu, \lambda)=(0, \pm k)$. The point $V$ where the dashed curves intersect, is the stationary point for the integrand and represents the ray from the vertex. The two points $S_{1}$ and $S_{2}$, where the dashed curves intersect the pole lines, correspond to singly diffracted edge rays (which will exist if $V$ lies to the left of the vertical pole line, respectively below the horizontal pole line). Additionally, there may be a contribution from $R$ representing the reflected ray, if $V$ lies below and to the left of the pole lines. The two points, $D_{1}$ and $D_{2}$, where the dashed curves intersect the branch lines, correspond to doubly diffracted edge rays (which will exist if $V$ lies to the left of the vertical branch line, respectively, below the horizontal branch line). It is clear from the geometry that only one doubly diffracted ray can exist for a particular $P$. The transition function required when $V$ is close to a pole line is the same [6] as used for the halfplane problem [1, Appendix]. When $V$ is close to a branch line, however, a different uniform expansion must be used [5, sect. 2.4]. Since the new transition functions, which only correct the field near the branch cut, are multiplicative, as is the one in [6], both will in general be applied simultaneously.

We now consider the situation, where $V$ lies close to $D_{1}$. The uniform expansion of (1) then becomes

$$
\begin{gathered}
\psi \sim e^{i\left(h\left(-k_{t 1}\right)+h\left(\lambda_{s}\right)\right) / 2} \\
\times\left\{A_{0} D_{-(1 / 2)}\left(t e^{-i(\pi / 4)}\right)+B_{0} D_{1 / 2}\left(t e^{-i(\pi / 4)}\right)\right\} \\
h(\lambda)=g\left(-\frac{x}{\rho_{1}} \sqrt{k^{2}-\lambda^{2}}, \lambda\right) \\
\rho_{1}=\sqrt{x^{2}+z^{2}}, \quad \lambda_{s}=-\frac{k y}{r} .
\end{gathered}
$$

Here, $r$ is the distance from $O$ to $P, D_{-(1 / 2)}$ and $D_{1 / 2}$ are the parabolic cylinder functions of order $-1 / 2$ and $1 / 2$, respectively, and the expressions for $t, A_{0}$ and $B_{0}$ are (notice printing error in [5, eq. (2.47a)]),

$$
\begin{gathered}
A_{0}=\sqrt{2 \pi} e^{-i(3 \pi / 8)} 2^{1 / 4} f\left(-k_{t 1}\right) \frac{\sqrt[4]{\Delta_{h}}}{\sqrt{\left|h^{\prime}\left(-k_{t 1}\right)\right|}} \\
B_{0}=\frac{\sqrt{2 \pi} e^{-i(\pi / 8)} 2^{-(1 / 4)}}{\operatorname{sign}\left(h^{\prime}\left(-k_{t 1}\right)\right) \sqrt[4]{\Delta_{h}}} \\
\times\left[\frac{f\left(\lambda_{s}\right)}{\left.\sqrt{\Delta_{\lambda}\left|h^{\prime \prime}\left(\lambda_{s}\right)\right|}-\frac{f\left(-k_{t 1}\right)}{\sqrt{\left|h^{\prime}\left(-k_{t 1}\right)\right|}}\right]}\right. \\
t=\sqrt{2 \Delta_{h}} \operatorname{sign}\left(h^{\prime}\left(-k_{t 1}\right)\right)
\end{gathered}
$$

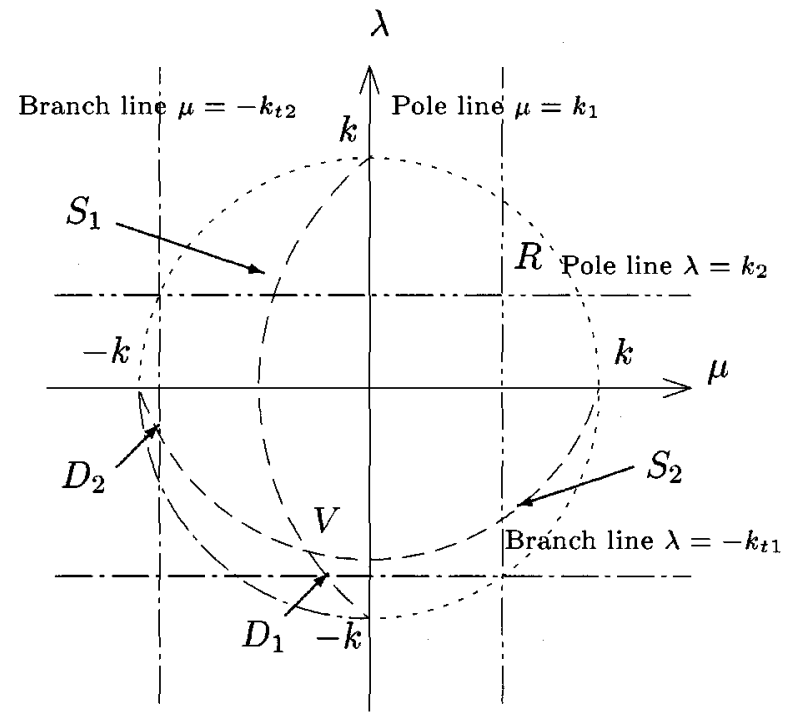

Fig. 2. Singularities for integrand.

with

$$
\begin{gathered}
f(\lambda)=F\left(-\frac{x}{\rho_{1}} \sqrt{k^{2}-\lambda^{2}}, \lambda\right) \sqrt{k_{t 1}+\lambda} \\
\times \frac{z \sqrt[4]{k^{2}-\lambda^{2}} \sqrt{2 \pi} e^{-i(\pi / 4)}}{\rho_{1} \sqrt{\rho_{1}}} \\
\Delta_{h}=\left|h\left(-k_{t 1}\right)-h\left(\lambda_{s}\right)\right|, \Delta_{\lambda}=\left|\lambda_{s}+k_{t 1}\right| .
\end{gathered}
$$

The situation considered corresponds to the ray picture shown in Fig. 1 and it is easily found that $h^{\prime}\left(-k_{t 1}\right)=-y_{0}$, where $y_{0}$ is the ordinate of the diffraction point $d_{1}$. When the parabolic cylinder functions in (7) are expanded asymptotically for large $|t|$, which physically corresponds to large $\left|y_{0}\right|$, and only the leading term of each function is retained, we obtain for $y_{0}<0$ (when the doubly diffracted ray does not exist)

$$
\psi \sim e^{i h\left(\lambda_{s}\right)} \sqrt{2 \pi} \frac{e^{-i(\pi / 4)} f\left(\lambda_{s}\right)}{\sqrt{L_{s}}}
$$

whereas for $y_{0}>0$

$$
\begin{aligned}
\psi \sim e^{i h\left(\lambda_{s}\right)} \sqrt{2 \pi} e^{-i(3 \pi / 4)} \frac{f\left(\lambda_{s}\right)}{\sqrt{L_{s}}} \\
+e^{i h\left(-k_{t 1}\right)} \sqrt{2 \pi} e^{-i(\pi / 4)} \frac{\sqrt{2} f\left(-k_{t 1}\right)}{\sqrt{\left|y_{0}\right|}}
\end{aligned}
$$

with

$$
L_{s}=\Delta_{\lambda}\left|h^{\prime \prime}\left(\lambda_{s}\right)\right|=\frac{r^{2}}{\rho_{1}^{2}}\left[\sqrt{y^{2}+\left(y_{0}^{2}-2 y y_{0}\right)\left(\frac{k_{1}}{k}\right)^{2}}-y\right] \text {. }
$$

The single term in (14) and the first term in (15) correspond to the vertex diffracted ray from $O$, whereas the second term in (15) corresponds to the doubly diffracted ray. 


\section{TRANSITION FUNCTIONS}

It is now possible to define a set of transition functions, that will neutralize the singularities in both the vertex ray and the doubly diffracted ray, occurring when $y_{0}=0$ as the ratio between the uniform ray expression and its asymptotic representation. The transition function for the vertex ray $F_{q v}$, assumes different forms for $y_{0}>0$ and $y_{0}<0$, while the transition function for the doubly diffracted ray $F_{q d d}$ only exists for $y_{0}>0$.

For $y_{0}<0, F_{q v}$ is obtained as the ratio between (7) and (14). The result is greatly simplified by assuming that $f()$ is slowly varying, so that $f\left(\lambda_{s}\right) \cong f\left(-k_{t 1}\right)$ since this allows us to eliminate $f($ from the transition function. The result, which is valid also when $V$ lies close to $D_{2}$, is conveniently expressed through two angles $\beta_{0}$ and $\beta_{1}$, where $\beta_{0}$ can equal $\beta_{0, x}$ or $\beta_{0, y}$, defined as the angle between the direction $\left(\theta_{0}, \phi_{0}\right)$ and the edge along $x$ or $y$, respectively, while $\beta_{1}$ can equal $\beta_{1, x}$ or $\beta_{1, y}$, defined as the angle between the vector from $O$ to $P$ and the edge along $x$ or $y$, respectively. If $V$ lies close to $D_{1}, \beta_{0, x}$ and $\beta_{1, y}$ apply; if $V$ lies close to $D_{2}, \beta_{0, y}$ and $\beta_{1, x}$ apply. With these definitions we have $y_{0}=r \cos \left(\beta_{0}+\beta_{1}\right) / \cos \beta_{0}$ and

$$
\begin{aligned}
& F_{q v}\left(\beta_{0}, \beta_{1}, r\right) \\
& =e^{-i \alpha_{1}}\left\{\alpha_{2} \alpha_{3} D_{-(1 / 2)}\left(\alpha_{2}^{2}\right)+\frac{1-\alpha_{3}}{\alpha_{2}} D_{1 / 2}\left(\alpha_{2}^{2}\right)\right\} \\
& \quad \beta_{0}+\beta_{1}>\frac{\pi}{2}
\end{aligned}
$$

where

$$
\alpha_{1}=\frac{1}{2} k r\left(1-\sin \left(\beta_{0}+\beta_{1}\right)\right), \quad \alpha_{2}=\sqrt[4]{4 \alpha_{1}} e^{-i(\pi / 8)}
$$

$$
\alpha_{3}=\sqrt{\left|\frac{\cos \beta_{0}\left(\sin \beta_{0}-\cos \beta_{1}\right)}{\sin ^{2} \beta_{1} \cos \left(\beta_{0}+\beta_{1}\right)}\right|} .
$$

In spite of appearances, the terms with $D_{-(1 / 2)}$ and $D_{1 / 2}$ are of the same order in $k$ and are therefore of equal importance.

For $\beta_{0}+\beta_{1}<(\pi / 2)\left(y_{0}>0\right)$ it is necessary to separate (7) into two expressions such that the first asymptotically equals (14), the second (15). Rewriting (7) as follows, adding and subtracting the term $A_{0} e^{-i(\pi / 2)} D_{-(1 / 2)}\left(-t e^{-i(\pi / 4)}\right)$ inside the parentheses leads to

$$
\begin{aligned}
& \psi \sim e^{i\left(h\left(-k_{t 1}\right)+h\left(\lambda_{s}\right)\right) / 2} \\
& \times\left\{\left[A_{0} e^{-i(\pi / 2)} D_{-(1 / 2)}\left(-t e^{-i(\pi / 4)}\right)\right]\right. \\
&+\left[A _ { 0 } \left(D_{-(1 / 2)}\left(t e^{-i(\pi / 4)}\right)-e^{-i(\pi / 2)}\right.\right. \\
&\left.\left.\left.\times D_{-(1 / 2)}\left(-t e^{-i(\pi / 4)}\right)\right)+B_{0} D_{1 / 2}\left(t e^{-i(\pi / 4)}\right)\right]\right\}
\end{aligned}
$$

where the term in the first bracket is the uniform expression for the vertex ray and the term in the second bracket is the uniform expression for the doubly diffracted ray. Taking the ratio between the uniform and nonuniform expressions, again assuming

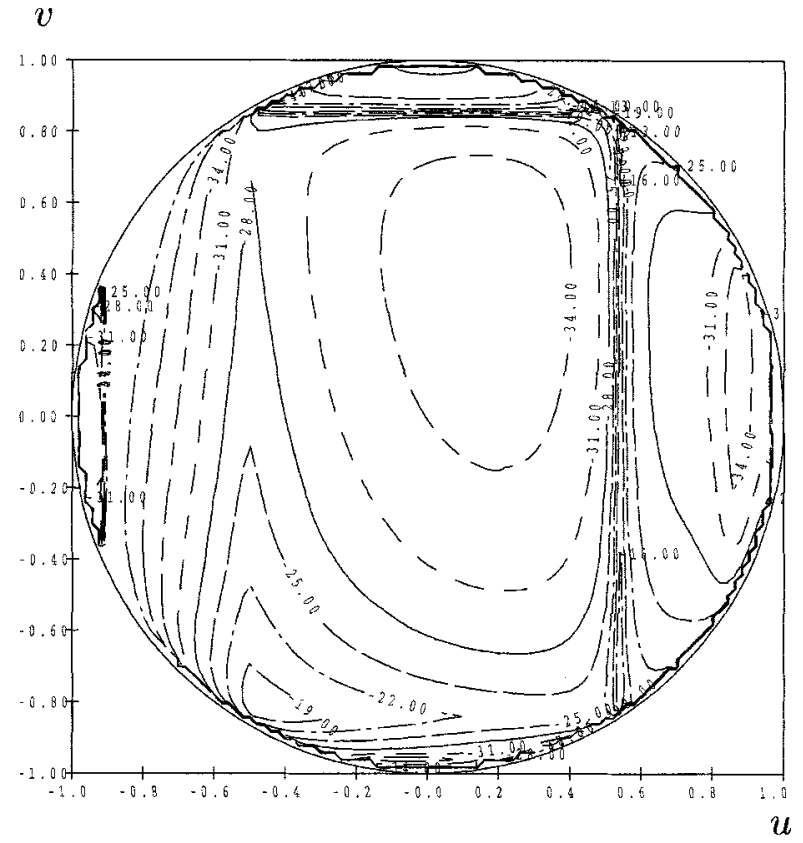

(a)

$v$

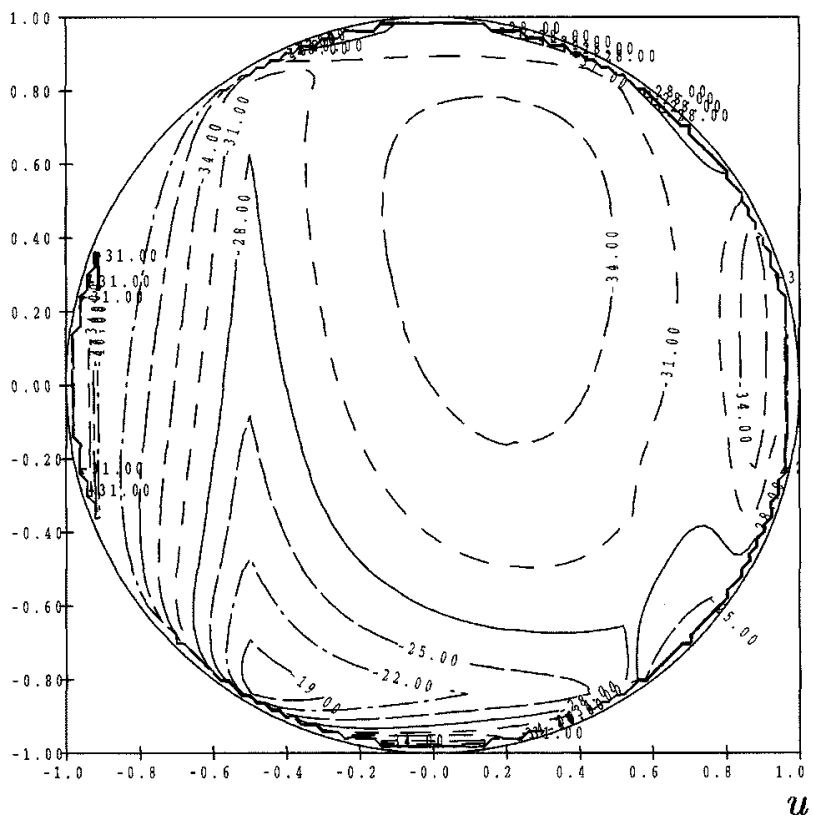

(b)

Fig. 3. Vertex ray plus doubly diffracted rays. (a) Without correction. (b) With correction.

$f\left(\lambda_{s}\right) \cong f\left(-k_{t 1}\right)$, the transition functions $F_{q v}$ and $F_{q d d}$ become

$$
\begin{gathered}
F_{q v}\left(\beta_{0}, \beta_{1}, r\right) \\
=e^{-i \alpha_{1}}\left\{\alpha_{2} \alpha_{3} D_{-(1 / 2)}\left(\alpha_{2}^{2}\right)-\frac{i\left(1-\alpha_{3}\right)}{\alpha_{2}} D_{1 / 2}\left(-\alpha_{2}^{2}\right)\right\} \\
\beta_{0}+\beta_{1}<\frac{\pi}{2} \\
F_{q d d}\left(\beta_{0}, \beta_{1}, r\right) \\
=e^{i \alpha_{1}} \frac{\alpha_{2}}{\sqrt{2}}\left\{D_{-(1 / 2)}\left(-\alpha_{2}^{2}\right)+i D_{-(1 / 2)}\left(\alpha_{2}^{2}\right)\right\} \\
\beta_{0}+\beta_{1}<\frac{\pi}{2} .
\end{gathered}
$$




\section{NUMERICAL RESULTS}

To illustrate the effect of the transition functions, Fig. 3 shows part of the diffracted field in the upper hemisphere with a source placed in $P=(3,5,1) \lambda$, plotted in a standard $u-v$ projection. Only the vertex ray, calculated using the diffraction coefficient $D_{V, H}$ defined in [1] and the doubly diffracted rays are included. In Fig. 3(a) the singularity at $u=0.535$ corresponds to the coalescence of $V$ and $D_{1}$ in Fig. 2, while the singularity at $v=0.862$ corresponds to the coalescence of $V$ and $D_{2}$. In Fig. 3(b) the (same) ray contributions have been corrected. The doubly diffracted ray that exists for $u>0.535$ is corrected with the factor $F_{q d d}\left(\beta_{0, x}, \beta_{1, y}, r\right)$, while the doubly diffracted ray that exists for $v>0.862$ is corrected with the factor $F_{q d d}\left(\beta_{0, y}, \beta_{1, x}, r\right)$. The vertex ray, which exists everywhere, is corrected with the factor $F_{q v}\left(\beta_{0, x}, \beta_{1, y}, r\right) F_{q v}\left(\beta_{0, y}, \beta_{1, x}, r\right)$. Since the transition functions have been defined such that they tend to unity away from the singularity they compensate, it is legitimate to multiply $D_{V, H}$ with two transition functions. Extensive calculations of the scattering from a hard, rectangular plate, for which a method of moments solutions can be found for comparison, has shown that the present theory is accurate down to levels of approximately $-30 \mathrm{~dB}$ for plate sizes above $14 \lambda$ (see [7]).

\section{CONCLUSION}

The paper presents transition functions for acoustical rays diffracted at the vertex of a hard quarterplane and for doubly diffracted edge rays. The results are obtained by a uniform asymptotic expansion of an exact expression for the scattered field. The transition functions ensure the accuracy of geometrical theory of diffraction calculations of acoustical scattering from hard, rectangular plates down to $-30 \mathrm{~dB}$. As a consequence, the application of the theory for scattering of electromagnetic fields around a quarterplane in [1] can be extended to any position of the dipole source.

\section{REFERENCES}

[1] N. C. Albertsen, "Diffraction by a quarterplane of the field from a halfwave dipole," Inst. Elect. Eng. Proc. Microwave Antennas Propagat., vol. 144, pp. 191-196, 1997.

[2] F. Capolino, S. Maci, R. Tiberio, and A. Toccafondi, "Uniform diffraction coefficients at a plane angular sector," in IEEE AP-S Symp. Dig., June 1994, pp. 586-589.

[3] F. Capolino and S. Maci, "Uniform high-frequency description of singly, doubly, and vertex diffracted rays for a plane angular sector," J. Electromagn. Waves Applicat.., vol. 10, pp. 1175-1197, 1996.

[4] J. Radlow, "Note on the diffraction at a corner," Arch. Rational Mech., vol. 19 , pp. 62-70, 1965.

[5] V. A. Borovikov, "Uniform stationary phase method," in IEE Electromagnetic Wave Series, ser. 40 London, U.K., 1994.

[6] R. G. Kouyoumjian and P. H. Pathak, "A uniform geometrical theory of diffraction for an edge in a perfectly conducting surface," Proc. IEEE, vol. 62, pp. 1448-1461, Nov. 1974.

[7] N. C. Albertsen, "A transition function for a vertex diffracted ray," in JINA Proc., 1998, pp. 445-448.

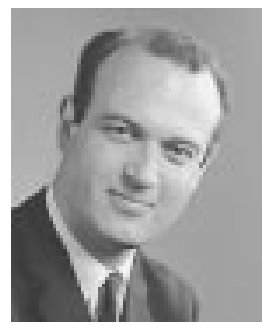

N. Chr. Albertsen was born in Frederiksberg, Denmark, in 1946. He recieved the M.Sc.E.E. and Ph.D. degrees from the Technical University of Denmark, Lyngby, in 1970 and 1975, respectively.

He has been Managing Director of TICRA since 1975 and an Associate Professor at the Technical University of Denmark since 1977, presently with the Department of Mathematical Modeling. His research interests include aperture antennas and diffraction theory.

Dr. Albertsen is a member of the Danish Academy of Technical Sciences and Chairman of the Danish Commission B of the International Union of Radio Science. 